\title{
Post-test probability that men in the community with raised plasma ferritin concentrations are hazardous drinkers
}

\author{
Hedley G Peach, Nicole E Bath
}

\begin{abstract}
Background-Raised plasma ferritin concentrations occur unexpectedly during iron studies done by primary care physicians. Plasma ferritin concentration has been positively associated with alcohol use among men.

Aim-To determine the post-test probability that men in the community with raised plasma ferritin concentrations are hazardous drinkers.

Methods-The subjects were 152 men, randomly selected from a city's electoral roll. Nineteen (12.5 (2.7)\%, mean (SEM)) admitted to drinking hazardously. The pretest probability of a man being a hazardous drinker was 0.125 . This was converted to pretest odds of 0.14 . The likelihood ratio (the ratio of the probability of obtaining a raised plasma ferritin concentration in a hazardous drinker (sensitivity) to the probability of obtaining a raised plasma ferritin concentration in a nonhazardous drinker (1-specificity)) was calculated for different plasma ferritin cut off points.
\end{abstract}

Results-A plasma ferritin level of $>652$ $\mu \mathrm{g} / 1$ gave the largest likelihood ratio, 4.16 . Post-test odds were obtained by multiplying the pretest odds $(0.14)$ by the likelihood ratio (4.16). A plasma ferritin level of $>652 \mu \mathrm{g} / 1 \mathrm{had}$ a post-test odds for a man being a hazardous drinker of 0.58 . This was converted to a post-test probability of 0.37 .

Conclusions-Inquiries could usefully be made into the alcohol consumption of men with a plasma ferritin concentration $>652 \mu \mathrm{g} / 1$, as approximately one in three would admit to drinking hazardously.

(f Clin Pathol 1999;52:853-855)

Keywords: ferritin; alcohol

The University of Melbourne, Ballarat Health Services Base Hospital, PO Box 577, Ballarat Victoria 3353, Australia

H G Peach

N E Bath

Correspondence to: Dr Bath email: a.temperley@ gpph.unimelb.edu.au

Accepted for publication 5 August 1999
A raised plasma ferritin concentration may be found unexpectedly during the course of iron studies by primary care physicians. Serum ferritin concentration has been positively associated with alcohol use among men in the community. Seventeen years ago, increased serum ferritin concentrations ( $>400 \mu \mathrm{g} / \mathrm{l}$ ) were found in $67 \%$ (SD $4.9 \%$ ) of middle aged men from the general population of Sweden who had serum $\gamma$-glutamyltransferase (GGT) values $>840$ IU, compared with only $2.5 \%$ of men with normal GGT values. ${ }^{1}$ More recently, a survey of employees of two large Australian corporations found that alcohol intake was one of the most important factors influencing the concentration of serum ferritin in men, although not in women. ${ }^{2}$ Heavy alcohol intake was associated with increased concentrations, even among men with normal levels of GGT.

A search of published reports did not reveal the post-test probability that men in the community with raised plasma ferritin concentrations were hazardous drinkers. When faced with a raised plasma ferritin concentration, it would be useful for primary care physicians to know the value above which it is most worthwhile enquiring into a patient's alcohol consumption. We have therefore determined the post-test probability that men in a regional Australian city with raised plasma ferritin concentrations are hazardous drinkers.

\section{Methods}

The sample comprised 152 men drawn from 163 who had participated in a cardiovascular disease risk factor prevalence survey in Ballarat and for whom frozen plasma was available for iron studies. The men from whom plasma was unavailable were similar to the others in age, number of drinks consumed a week, and proportion of hazardous drinkers. Two hundred and forty two men, randomly selected from the electoral roll, had been invited to participate in the cardiovascular survey, giving a $67(3.0) \%$ response (mean (SEM)), which is usual and acceptable for surveys of the general population.

Data on alcohol consumption had been collected through a self administered questionnaire. ${ }^{3}$ Participants were asked about the number of days on which alcohol was usually consumed in a week and the number of drinks on a drinking day. Types of alcoholic beverage consumed were recorded, but life time drinking histories were not. Frozen plasma samples were retrieved and thawed. The concentrations of ferritin and transferrin were measured using an immunoturbidimetric assay (Boehringer Mannheim).

Subjects were classified as drinking hazardously if the average daily consumption was reported to be at least five standard drinks (50 grams of alcohol). ${ }^{3}$ The average daily alcohol consumption and plasma ferritin concentrations of hazardous and non-hazardous drinkers were compared using a one way analysis of variance on logarithmically transformed data.

The likelihood ratio, the ratio of the probability of obtaining a raised plasma ferritin concentration in a hazardous drinker (sensitiv- 
ity) to the probability of obtaining a raised plasma ferritin concentration in a nonhazardous drinker (1-specificity), ${ }^{4}$ was calculated for different plasma ferritin cut off points. The post-test odds of a man in the community with a plasma ferritin concentration above a cut off point being a hazardous drinker were obtained by multiplying the pretest odds of a man being a hazardous drinker by the likelihood ratio. ${ }^{4}$ The pretest odds were obtained by converting the pretest probability (prevalence of hazardous drinking in the community) into odds. ${ }^{4}$

The study was approved by the Ballarat Base Hospital ethics committee.

\section{Results}

The average age of the subjects was 45.4 years. Plasma ferritin concentrations ranged from 14 to $1703 \mu \mathrm{g} / 1$. As others have found, ${ }^{2}$ plasma ferritin concentrations were positively correlated with the daily amount of alcohol consumed in grams, both in total $(p=0.016)$ and from beer in particular $(\mathrm{p}<0.001)$, but not from wine or spirits.

The prevalence (mean (SEM)) of hazardous drinking was $12.5(2.7) \%$. The average age of the hazardous drinkers, 41.5 years, was not significantly different from that of the nonhazardous drinkers, 45.9 years. Compared with non-hazardous drinkers, men who drank hazardously had a greater average daily consumption of alcohol in grams from spirits (1.93 v $0.62, \mathrm{p}=0.0007)$ and wine $(6.30 v 2.15$, $\mathrm{p}=0.010)$, but particularly from beer $(24.0 \mathrm{v}$ $3.15, \mathrm{p}<0.001)$. The average plasma ferritin concentration of the men who drank hazardously $(443.2 \mu \mathrm{g} / \mathrm{l})$ was significantly greater than that of non-hazardous drinkers $(254.1 \mu \mathrm{g} / \mathrm{l})$ $(\mathrm{p}<0.001)$.

The pretest probability that a man was a hazardous drinker was 0.125 . This converted to pretest odds of $0.14(0.125 / 1-0.125)$.

The upper limit of the laboratory's normal range for plasma ferritin concentration was 300 $\mu \mathrm{g} / 1$. In all, 29 (3.7)\% of subjects had a plasma ferritin concentration above normal. They included both hazardous and non-hazardous drinkers. A plasma ferritin concentration of $>300 \mu \mathrm{g} / \mathrm{l}$ gave a likelihood ratio of 1.55 . A plasma ferritin concentration of $>300 \mu \mathrm{g} / 1 \mathrm{had}$ a post-test odds of a man being a hazardous drinker of $0.22(0.14 \times 1.55)$.

A plasma ferritin concentration of $652 \mu \mathrm{g} / 1$ gave the largest likelihood ratio, 4.16. Five per cent of subjects had a plasma ferritin concentration $>652 \mu \mathrm{g} / \mathrm{l}$. A plasma ferritin concentration $>652 \mu \mathrm{g} / \mathrm{l}$ had a post-test odds of a man being a hazardous drinker of $0.58(0.14 \times$ 4.16). This converted to a post-test probability of $0.37(0.58 / 1+0.58)$.

Two subjects had a plasma ferritin concentration $>1000 \mu \mathrm{g} / 1$ and a transferrin saturation $>60 \%$, suggesting possible haemochromatosis. One, with a transferrin saturation of $61 \%$ and a plasma ferritin concentration of $1476 \mu \mathrm{g} / \mathrm{l}$, drank five to eight standard drinks every day. The other, with a transferrin saturation of $95 \%$ and a plasma ferritin concentration of 1164 $\mu \mathrm{g} / \mathrm{l}$, was a man who drank on less than one day a week and consumed only one or two drinks on a drinking day, but had a father who died of liver cancer aged 62. None of the other participants had a family history of haemochromatosis suggesting heterozygosity for this condition or porphyria cutanea tarda.

\section{Discussion}

Twenty nine per cent of the men had a plasma ferritin concentration above the laboratory's normal range. However, the plasma ferritin level corresponding to the largest likelihood ratio (probability of obtaining a raised plasma ferritin concentration in a hazardous drinker to that of obtaining a raised concentration in a non-hazardous drinker) was $652 \mu \mathrm{g} / \mathrm{l}$. In this population where the pretest probability of hazardous drinking was 0.13 , the post-test probability that a man in the community with a plasma ferritin concentration $>652 \mu \mathrm{g} / \mathrm{l}$ was a hazardous drinker was 0.37 . It follows that our primary care physicians should ask such men about their alcohol consumption, because approximately one in three will admit to drinking hazardously and can be offered counselling. Unlike the likelihood ratio, the post-test probability that a man with a plasma ferritin concentration $>652 \mu \mathrm{g} / \mathrm{l}$ is a hazardous drinker will vary with the prevalence of hazardous drinking in the community and hence with the pretest probability. The pretest probability of hazardous drinking in our population, 0.13 , was similar to the pretest probabilities of 0.09 and 0.11 found among men in other community studies. ${ }^{56}$ Our conclusions are therefore applicable to primary care physicians in other populations.

The reliability of quantitative questionnaires inquiring after the frequency of alcohol consumption in the general population is well established. The questionnaire employed in this study was that used by the National Heart Foundation of Australia in its prevalence surveys of cardiovascular disease risk factors among adults in Australia's capital cities. ${ }^{3} \mathrm{Haz}-$ ardous drinkers were more likely to be smokers and single or divorced, showing that the questionnaire had identified a similar subgroup of the population as had other questionnaires used overseas. ${ }^{7}$

The relation between alcohol consumption and serum ferritin concentration has been investigated in many countries. Some studies have published the actual proportion of people abusing alcohol who have a serum ferritin concentration above normal. Raised serum ferritin concentrations have been found in $37(5.7) \%$ of male alcoholics in ambulatory care in Sweden, ${ }^{8}$ in 58 (4.7)\% of patients with alcoholic liver disease in Norway, ${ }^{9}$ in 5 (1.7)\% of patients (mostly men) admitted to an alcohol withdrawal unit in England, ${ }^{10}$ and in 67 (4.9)\% of middle aged men from the general population of Sweden who were heavy drinkers. ${ }^{1}$ In these studies, serum ferritin concentrations were regarded as raised if they exceeded $240,200,1000$, and $400 \mu \mathrm{g} / 1$, respectively. Although raised serum ferritin concentrations have been found in $16-67 \%$ of heavy drinkers, this is the first time that the post-test 
probability of men in the community with raised plasma ferritin concentrations being hazardous drinkers has been calculated. The percentage of haemoglobin comprising Hba $1 \mathrm{a} 2$ or HbA1d 1 is more sensitive at distinguishing between social and heavy drinkers than a raised plasma ferritin concentration, at least among women, but these values are not measured routinely. ${ }^{11}$

Dorevitch Pathology, Melbourne, conducted the iron studies.

1 Kristenson H, Fex G, Trell E. Serum ferritin, gammaglutamyltransferase and alcohol consumption in healthy glutamyltransferase and alcohol consumption in heal
middle aged men. Drug Alcohol Depend 1981;8:43-50.

2 Leggett BA, Brown NN, Bryant SJ, et al. Factors affecting the concentrations of ferritin in serum in a healthy Australian population. Clin Chem 1990;36:1350-5

3 National Heart Foundation. Risk factor prevalence study. Survey No 3. Canberra: National Heart Foundation, 1989.
4 Motulsky H. Intuitive biostatistics. Oxford: Oxford University Press, 1995:136-7.

5 Whitehead TP, Clark CA, Whitfield AGW. Biochemical and haematological markers of alcohol intake. Lancet 1978;i: 978-81.

6 Shaper AG, Pocock SJ, Ashby D, et al. Biochemical and haematological response to alcohol intake. Ann Clin Biochem 1985;22:50-61.

7 Seppa K, Laippala P, Saarni M. Macrocytosis as a consequence of alcohol abuse among patients in general consequence of alcohol abuse among patien
practice. Alcohol Clin Exp Res 1991;25:871-6.

practice. Alcohol Clin Exp Res 1991;25:871-6.
8 Lundin L, Hällgren R, Birgegård G, et al. Serum ferritin in alcoholics and the relation to liver damage, iron state and erythropoietic activity. Acta Med Scand 1981;209:327-31.

9 Bell H, Skinningsrud A, Raknerud N, et al. Serum ferritin and transferrin saturation in patients with chronic alcoholic and non alcoholic liver diseases. F Intern Med 1994;236: $315-22$.

10 Ford C, Wells FE, Rogers JN. Assessment of iron status in association with excess alcohol consumption. Ann Clin Biochem 1995;32:527-31.

11 Hurme L, Seppa K, Rajaniemi H, et al. Chromatographically identified alcohol-induced haemoglobin adducts as markers of alcohol abuse among women. Eur F Clin Invest 1998;28:87-94. 